EOMmunutriln? Communication et organisation

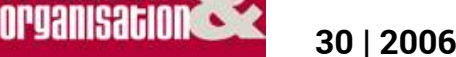

Modèles et modélisations, pour quels usages ?

\title{
Les modèles de la communication et leur objet d'étude
}

Miguel de Aguilera

\section{(2) OpenEdition}

1 Journals

Édition électronique

URL : http://journals.openedition.org/communicationorganisation/3457

DOI : 10.4000/communicationorganisation.3457

ISSN : $1775-3546$

Éditeur

Presses universitaires de Bordeaux

Édition imprimée

Date de publication : 1 octobre 2006

Pagination : 110-125

ISSN : 1168-5549

\section{Référence électronique}

Miguel de Aguilera, «Les modèles de la communication et leur objet d'étude », Communication et organisation [En ligne], 30 | 2006, mis en ligne le 21 juin 2012, consulté le 04 mai 2019. URL : http:// journals.openedition.org/communicationorganisation/3457 ; DOI : 10.4000/

communicationorganisation.3457

Ce document a été généré automatiquement le 4 mai 2019.

(C) Presses universitaires de Bordeaux 


\title{
Les modèles de la communication et leur objet d'étude
}

\author{
Miguel de Aguilera
}

1 Les activités professionnelles que l'on classe d'ordinaire dans la catégorie « communication » constituent un ensemble de pratiques professionnelles de différentes natures allant de la production de contenus à la recherche scientifique. Chacune d'entre elles, toujours rattachées à un cadre institutionnel ou organisationnel, s'appuie, de manière plus ou moins explicite, sur une suite d'hypothèses, de croyances, d'idées qui incluent dans tous les cas, parmi d'autres éléments, une représentation simplifiée de l'objet de cette activité, c'est-à-dire, un modèle de communication et qui, dans une certaine mesure, ne sont que la concrétisation de certains modes de penser la communication en fonction de son contexte de production. Car nous sommes des sujets historiques qui, au fil de nos activités, ne pouvons nous soustraire entièrement à l'influence de contingences environnementales. Parmi celles-ci on inclut certaines formes dominantes de concevoir et de comprendre nos actes; la traduction dans la sphère scientifique en étant l'adoption de certaines philosophies des sciences et partant de postures épistémologiques et méthodologiques. Ces postures, à leur tour, définissent l'activité traitée ; ce qui comprend des questions portant sur l'objet d'étude, sur la manière d'aborder l'examen de ces phénomènes et, même, dans quel but doit-on le faire, par qui, et dans quelles conditions, ce qui implique le cadre institutionnel de sa réalisation.

2 La reconstruction succincte de l'ensemble des cadres théoriques qui ont guidé l'étude scientifique de la communication est une tâche complexe et risquée (Miège, 2004). Ceci, surtout, parce que la communication est un domaine polymorphe qui recouvre une gamme très étendue, voire croissante, d'activités humaines et qui, en conséquence, a réuni autour d'elle une longue liste d'acteurs sociaux et donc autant de points de vue ou de «logiques». Même en réduisant le champ de ces activités à celui qui est traditionnellement admis comme propre à la communication, constitué autour d'un objet d'étude déterminé écartant un grand nombre de modalités communicationnelles, la tâche n'est pas facile. En effet, à côté d'un modèle dominant et de quelques autres tout aussi éminents, coexistent différentes approches ayant atteint une égale acceptation, autant 
d'approches nécessaires à l'étude des aspects concrets de la communication humaine. Pourtant, celui qui entreprendrait cette reconstruction succincte des cadres devra assumer sa nécessaire subjectivité et suivra dans cette narration un fil conducteur singulier ayant comme principal argument l'objet d'étude défini pour la communication. En effet, en science de la communication, d'un point de vue historique, une définition a dominé : elle est même parvenue à orienter et à en articuler parfois les positions critiques. Elle prend ses références essentielles dans l'une des modalités qu'adopte la communication et elle tend à satisfaire les objectifs principaux déterminés par le contexte social dans lequel cette activité scientifique se produit. Voilà pourquoi le modèle de science de la communication ainsi établi n'est pas apte à comprendre correctement certaines des formes de communication les plus caractéristiques de la société actuelle: par exemple, et surtout, ce que l'on connaît comme « culture populaire » ou « de masse ».

En conséquence, cet article traitera en premier lieu certaines des causes - au niveau des concepts - qui déterminent la définition de l'objet d'étude de la communication et qui rendent difficile la pleine compréhension d'autres objets, et en particulier celui que constitue la culture populaire. Je m'attacherai ensuite à l'objet d'étude dominant de cette science et surtout à son élément de base : l'information. En dernier lieu, je rendrai compte d'apports théoriques plus récents qui contribuent de manière décisive à modifier cet objet d'étude et plus généralement le modèle de compréhension des sciences de la communication.

\section{La communication, entre (l')« être » et( le) « devoir être »}

4 Même si on trouve quelques précédents dans l'histoire, c'est à la fin des années trente que les premières tentatives sérieuses entreprises pour une étude scientifique de la communication afin d'en comprendre les processus et en orienter les pratiques furent réalisées. Et c'est à la fin de la seconde guerre mondiale que les premiers postulats solides virent le jour. Ceci n'est pas, bien évidemment, le fruit du hasard mais celui d'un concours de circonstances propices, dont la plus évidente est l'installation progressive d'un système de communication centré sur la télévision. Outil qui, outre une dimension commerciale remarquable instaura une visibilité nouvelle, généra des flux d'information abondants qui ont proposé au citoyen une vision de la réalité présentée comme un univers bigarré de symboles configurés selon des matrices narratives et, qui a recueilli (en tout lieu et à toutes les périodes) les fantasmes de millions de personnes. L'établissement dudit système constitue, à son tour, une manifestation marquante d'une société orientée pleinement vers la création d'un réseau complexe de communication de personnes, de biens et d'idées.

C'est bien qu'en fin de compte, aucun système de communication humaine ne peut être compris sans interroger le contexte social dans lequel il naît et se développe (de Aguilera, 1989, 63). Dans ce cas précis, ce qui éclaire ce système de communication et lui donne un sens, c'est la dénommée "société industrielle », qui s'enracine dans le projet "éclairé " de la Modernité. Ainsi, pour une exacte compréhension du phénomène faudrait-il examiner la conjonction d'une série de facteurs, de conditions et d'agents qui l'ont rendu possible, c'est-à-dire l'addition de différentes causes (raisons explicatives) tant matérielles qu'« idéales » selon l'expression wéberienne. De nombreux auteurs, on le sait, 
ont examiné les relations de chacune d'elles avec le développement de ce système de communication dans nombre d'ouvrages. Parmi ceux qui traitent du second type de causes, les «idéales", on mentionnera le livre d'Armand Mattelart qui s'attache à reconstruire le processus d'attribution des principales acceptions du terme communication tel qu'on l'entend aujourd'hui. (Mattelart, 1995). Cet auteur reconnaît entre autre l'influence fondamentale des églises chrétiennes sur le domaine culturel associé au terme communication. Il souligne également sa pleine inclusion dans le projet de la Modernité : la communication conçue comme "projet et implantation de la raison " (Mattelart 1995 : 19), inscrite dans un idéal plus large de compréhension et de domination du monde et de n'importe quelle circonstance qui y concourt par le raisonnement humain.

Cet auteur est, sans nul doute, dans le vrai à la fois en posant les fondements « idéaux » de la communication comme base de pratique professionnelle et objet d'étude scientifique et, en structurant son «archéologie des savoirs sur la communication» autour de quatre processus intellectuels parallèles (Mattelart, 1995 : 13). Il est néanmoins surprenant qu'un ouvrage guidé par cette intention ne prenne pas en compte un autre facteur crucial étudié dans un travail précédent. Il y soulignait, s'agissant des travaux d'ADORNO, «la méfiance éclairée " de ce dernier et de nombreux auteurs face à certaines formes culturelles basées sur leur reproductibilité technique, pour reconnaître ensuite que de " ce vigoureux acte de foi sur la valeur de la haute culture naîtront de nombreux malentendus " (Mattelart, 1993 : 221).

7 Toutefois, le philosophe Gustavo Bueno donne une représentation claire du rôle que joue la culture dans la Modernité et partant, dans le type de société où elle est née. En effet, dans un de ses ouvrages les plus connus, il se propose de démythifier la culture car, selon lui, la modernité n'a pu faire autrement que de fonder son projet "éclairé » sur des mythes comme la Liberté, la Richesse, le Bonheur, l'Egalité, la Démocratie et, en ce qui nous concerne, la Culture (Bueno, 1996: 11). Ils eurent tous une fonction sociale en participant d'un processus de sécularisation qui substitua l'univers des idées religieuses en vigueur pendant des siècles par un autre dont la pierre angulaire serait la raison humaine. La culture, concrètement, a signifié la sécularisation du mythe médiéval de la Grâce de Dieu : si auparavant ce qui différenciait l'homme de l'animal était la conscience, basée sur l'âme qu'inondait la grâce divine, la culture représenterait maintenant le support de cette conscience et, sa sédimentation, le résultat de l'action rationnelle exercée durant des siècles par des collectivités humaines, reflétant ainsi, en termes séculiers, l'« âme d'un peuple ».

8 Mais selon lui, la culture acquiert des traits de mythe obscurantiste car lorsque la Modernité rationalise ce mythe, parmi d'autres, et le dépouille en apparence de sa condition originelle de fable et d'illusion, il le transforme en une sorte de "récit suprarationnel » (Bueno, 1996 : 26), en idée-force qui en guidant l'action des sujets sociaux jette les bases de certaines formes de vie en société et, leur donne sens. Mais son objectivation induit aussi des champs d'action spécifiques qui le relie à des rites, des normes, etc.... Dans le cas de la culture, on a distingué trois domaines d'action principaux, l'un encensé, l'autre méprisé, et pour être précis, celui que la science de la communication aura avant tout à étudier. Dans ce processus de mythification moderne de la culture, on a donc distingué trois niveaux d'expression de celle-ci qui correspondent simplement à des niveaux de pratiques culturelles: la haute culture, celle des élites, restreinte dans son accès et sa jouissance (copies uniques, théâtres et rituels), la culture 
populaire ou folklorique (Volks, folk) celle des traditions du peuple, et la culture de masse (techniquement (re)produite, marchandisée et standardisée) (de Aguilera, 2004: 150). Quelques spécialistes ont précisé récemment ce schéma en remplaçant par populaire (Real, $2001: 168$ ), le concept confus et galvaudé de « masse ».

Le mythe de la culture, dans ses modalités et ses pratiques, a eu des effets pervers sur la culture des classes populaires urbaines. Il est, en tout cas, à l'origine de sévères critiques engendrées entre autres par une marchandisation marquée dont l'aboutissement serait la production de contenus de "qualité médiocre ", destinés à satisfaire le goût culturel d'un large public, majoritairement peu qualifié, c'est à dire, la masse. Par son origine, cette culture populaire ou de masse, produite et diffusée par les industries culturelles grâce à la " communication de masse ", ne relèverait pas du domaine de la culture traditionnelle le locus idéal du milieu rural-; elle ne répondrait pas non plus au positionnement des élites cultivées, entre autres raisons, parce qu'elle ne fait pas appel à la raison mais à l'émotion (ou, si on préfère, à l'éros freudien). Le mythe de la culture était ainsi déplacé au champ de la communication car d'évidence, la communication consiste essentiellement en manifestations pratiques de la culture dans laquelle baignent les acteurs sociaux. Mais, à tenir compte de la conception «idéale », le type de culture à étudier par la science de la communication serait d'emblée disqualifiée rendant ainsi sa juste compréhension plus difficile. A savoir que, aussi bien le mythe de la culture que le mythe conséquent et parallèle de la communication sont le fondement du discours public qui critique sévèrement d'un point de vue moral (en se plaçant au niveau du « doit être »), les pratiques culturelles de la grande majorité de la population. On notera qu'il en est de même de la pratique scientifique qui les développe et les légitime. Pourtant, depuis plusieurs décades, nous consommons, le plus souvent apprécions et intégrons dans notre vie quotidienne des produits fabriqués par les industries culturelles. Mais quand le discours public, qui recrée certains fondements idéaux de notre vie collective, les rejette, que les orientations scientifiques dominantes les méprise, on renonce à les comprendre rationnellement (pourquoi font-elles partie de notre vie quotidienne malgré le rejet public ?) et y compris à les orienter dûment si nécessaire ; on rend ainsi plus aisé la " colonisation mercantile» de nos désirs et de nos plaisirs que les industriels, eux, reconnaissent, comprennent bien et travaillent à satisfaire.

Même si, paradoxalement, elle n'a pas fait partie du champ d'étude traditionnel de la communication, le cas de la musique en est une bonne illustration. En effet, cette pratique culturelle qui en appelle aux émotions a connu un singulier processus de mythification, car on a pu distinguer dans le domaine musical les trois catégories qui organisent la culture.

11 La musique populaire, distribuée par de puissantes industries aux fabuleuses affaires, est présente dans la vie quotidienne d'une part considérable de la population; malgré cela, peu nombreux sont les chercheurs ayant étudié cette pratique culturelle. La musique folklorique, quant à elle, a bénéficié de la considération publique et soulevé l'intérêt, au fil du temps, d'un nombre croissant de chercheurs. Mais l'objet traditionnel, et longtemps exclusif, d'étude des spécialistes, bien qu'étant une pratique culturelle d'une part réduite de la population est la « musique savante » ou « musique classique».

12 Au-delà de leur qualité indiscutable, il est paradoxal que l'on considère « classiques » ces formes musicales nées d'un contexte spatio-temporel déterminé : celui des sociétés au développement industriel vigoureux. Ces formes musicales, comme toute autres, sont le résultat du contexte dans lequel elles se sont développées et partant reflètent certaines 
de leurs caractéristiques : par exemple, le développement de la subjectivité bourgeoise ou la domination de la femme par l'homme ${ }^{1}$. Mais la musique classique a connu un processus singulier de mythification, qui repose, entre autres, sur la sécularisation d'une certaine tradition «essentialiste » attribuée à la musique et à l'expérience musicale dont le " mythe de Beethoven » est l'illustration. (Cook, 2003 : 44). L'établissement de certains canons esthétiques et d'un « répertoire » de musique de qualité, que l'on considérerait de ce fait classique, ajoutés à l'institutionnalisation des acteurs de ce domaine musical et, des rituels par eux observés en fut la traduction. De même, l'institutionnalisation d'un "modèle marchand» basique qui repose sur l'exploitation des droits d'auteur. Essentiellement, l'«œuvre» créée par le compositeur est aussi bien exploitée par l'éditeur des partitions que par le promoteur et l'interprète qui la joue devant un auditoire payant sur une scène donnée.

Ce modèle d'exploitation du phénomène musical dans la société bourgeoise a été développé ultérieurement par les industries culturelles qui, grâce à certaines médiations techniques et diverses circonstances, vont engranger de considérables bénéfices en fournissant de la musique au public pour les différents moments de sa vie quotidienne. Il en va, certes de même et en des termes semblables, dans nombre de domaines de l'activité culturelle. Ces derniers ont également connu un processus d'institutionnalisation qui, entre autres spécificités, comprend un modèle d'exploitation basique grâce auquel certaines industries, avec l'appui de médiations techniques -aptes à la production, à la diffusion et à la reproduction- découvrent des éléments culturels dont l'originalité permet la mise en place de droits d'auteur et un traitement qui les rendent suffisamment attractifs pour qu'un grand nombre de récepteurs leur consacre loisir, énergie, temps, argent. Il comporte aussi la détermination des principaux acteurs susceptibles de participer à ces processus ainsi que les modalités de leur participation.

\section{L'information organise le modèle}

14 L'institutionnalisation de la communication a englobé ses différents aspects, y compris la matière que l'on devait traiter scientifiquement (son objet d'étude), ainsi que les modalités et les acteurs aptes à l'accomplir. C'est ainsi que, suite à la multiplication de la production et de la diffusion des messages et au développement des industries culturelles, il a fallu délimiter rapidement un domaine d'intervention pour les spécialistes susceptible de fournir les données destinées à satisfaire les besoins industriels et, de manière plus générale, de traiter les possibilités quasi thaumaturgiques attribués aux "puissants " mass-média. Même si, des professionnels venus de divers horizons du savoir ont contribué à couvrir ce domaine professionnel, il prend sa source, surtout à ses débuts, avant que l'on ne reconnaisse le domaine particulier de ceux que d'aucuns appellent "communicologues", dans deux domaines spécialisés respectivement dans la connaissance et le traitement de l'esprit humain et des grands groupes sociaux, c'est-àdire, la psychologie et la sociologie.

Toutefois, dans un premier temps, la détermination de ce champ d'étude a fait l'objet, au départ, de certaines polémiques. Certains chercheurs ont proposé que cette science, étant donné la centralité de ces phénomènes dans divers aspects de notre vie en société, traite des complexités de la communication humaine dans ses différentes manifestations, d'où l'intérêt d'une approche interdisciplinaire. D'autres, à leur tour, ont voulu centrer l'étude de la communication sur sa relation avec la subjectivité humaine. Mais, face à ces 
propositions, très vite s'est imposée la conception qui centrait l'étude de la communication humaine essentiellement sur les messages et en particulier les messages à caractère informatif, portés par les médias et sur les effets quasi thaumaturgiques produits sur les auditoires d'individus isolés mais composant d'énormes masses anonymes hétérogènes et dispersées sur des territoires étendus.

Parmi les circonstances contextuelles qui ont contribué à fixer cet objet d'étude, il convient de mentionner certaines conséquences de la Seconde Guerre Mondiale ${ }^{2}$ ainsi que les exigences posées par le modèle d'exploitation de ces médias (qui, parmi d'autres questions, avaient à mesurer quelques variables intervenant sur la communication) (Ang, 1996). L'étude des communications autour de ce premier objet a été effectuée par des chercheurs issus des deux courants méthodologiques dominants à cette époque -même si, entre l'un et l'autre, les différences sont bien sûr importantes. Le premier, en vigueur dans les pays du bloc soviétique: le matérialisme historique, utilisant surtout des procédés qualitatifs, cherchait à démontrer d'une part la validité des assertions de $\mathrm{K}$. Marx, F. Engels et des auteurs du même courant de pensée, d'autre part, la preuve du bien-fondé des pratiques communicationnelles utilisées par les élites gouvernantes en vue de développer la société socialiste. (Manaev, 2003). Cette orientation méthodologique a, cependant, récolté ses fruits les plus importants en s'appliquant à l'étude de certains aspects des communications propres aux pays occidentaux; en effet, aussi bien la vigoureuse critique de la culture et de la communication réalisée dans le cadre de l'École de Frankfort que son développement ultérieur par le courant centré sur l'économie politique de la communication ont mis l'accent sur l'influence qu'exerceraient sur les travailleurs les messages élaborés et diffusés par les mass médias toujours en adéquation avec les intérêts économiques et idéologiques des classes dominantes.

17 L'autre courant correspondrait mieux au mode de compréhension des sciences humaines et sociales du bloc conduit par les États Unis : le positivisme. Ce dernier, dans l'étude scientifique de la communication, sous l'influence principale de la psychologie behaviouriste et de la sociologie fonctionnaliste, se développera sur une ligne identifiée par plusieurs appellations génériques, telles que "Mass Communications Research", "recherche administrative», bien qu'il me semble plus adéquat de la qualifier d'« informationnelle» (del Río, 1996: 42/ Dahlgren, 1998: 44), surtout si l'on prend en compte le rôle crucial que le concept d'information a joué dans ce courant tout au long de ces années. En même temps, le développement de la connaissance scientifique et technologique survenu après la Seconde Guerre Mondiale a, avec d'autres circonstances, accru considérablement le rôle de l'information à différents niveaux.

Par conséquent, une telle cette étude, conformément à son orientation méthodologique et aux raisons qui l'expliquent, a établi rapidement un modèle linéaire, à tendance

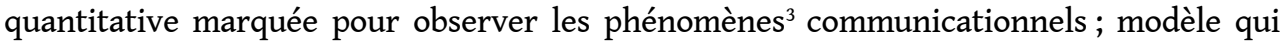
répond tout spécialement au point de vue de ceux qui produisent les contenus, s'est d'abord attaché au travail des émetteurs pour ensuite, grâce à des médiations technologiques déterminées, élaborer et diffuser des messages qui arriveraient aux récepteurs, pris comme individus isolés, sans la moindre référence à leur position sociale, et produiraient certains effets sur eux. De ce point de vue l'information apparait déjà comme principe organisateur, considérant les media en tant que diffuseurs de messages (pris comme éléments manifestes et mesurables dont on ignore les contextes discursifs) qui auraient un impact sur les pensées et les actes d'individus composant une audience de masse ; l'impact de ces messages serait la cause nécessaire et suffisante pour intervenir 
sur les attitudes et comportements de cette audience. Quand les premières formulations de cette direction d'étude commencent d'être posées -s'étendant aussi, soit dit en passant, à d'autres modalités, non informationnelles, de la communication-, les circonstances du contexte social d'après-guerre, la consolident et lui donnent un nouvel élan, tout en la modifiant en même temps; circonstances telles que la convergence d'une série de développements scientifiques qui pour l'essentiel répondaient à ce même point de vue. Ainsi, la théorie de l'information, la cybernétique, la théorie générale des systèmes, le structuralisme, l'intelligence artificielle et même la biologie cellulaire ont été les composantes principales du contexte intellectuel de cette période (Burnett y Marshall, 2003/ Gere, 2002) - et perdurent encore de nos jours avec une indubitable force. Dans tous les cas, l'information s'est imposée comme l'un des concepts centraux, l'un des principes organisateurs de recherches scientifiques à cette période. Il n'apparaît pas nécessaire d'approfondir ces idées, largement connues, ni de souligner davantage l'importance de ces postulats et de leur influence sur notre société, importance accrue si on y ajoute d'autres principes et centres d'intérêt traditionnels -comme la recherche de machines intelligentes et leur rapport direct à la raison instrumentale (analysée avec succès par plusieurs membres de l'École de Frankfort). Cette série de postulats, avec l'information comme pierre angulaire, s'est avérée utile dans la société d'après-guerre en devenant le principe organisateur de la technologie, de la stratégie militaire, de l'industrie et des affaires, etc. - et, plus encore dans son développement, pour le modèle social de nos jours.

C'est en intégrant ces lignes de pensée -généralement concordantes entre elles et avec des traditions bien ancrées dans notre société et les intérêts de certains groupes sociaux que le courant d'étude dont je fais mention-s'est consolidé, atteignant une situation hégémonique qui devait influencer la science de la communication dans son ensemble. Elle a donc étudié principalement les messages transmis par les mass médias -bien qu'actuellement elle couvre aussi ceux transmis par d'autres moyens de communication. Et elle a attaché un intérêt particulier aux effets de natures diverses, qu'ils proviennent de contenus d'information, de loisir ou mêmes commerciaux, que ces messages ont sur leurs récepteurs. L'objet d'étude défini par ce courant, étendu à l'ensemble de la science de la communication, est en bonne partie conditionné par une série d'éléments, matériels et idéaux, propres au milieu dans lequel il s'est développé ; parmi les idéaux, en somme et avant tout, par deux mythes : celui de la culture et celui de la technologie (Vitalis, 1999 : $40)^{4}$. Cet objet d'étude, adapté à une orientation méthodologique qui présente de sérieuses limitations, n'est pas adéquat pour examiner avec justesse les communications humaines et leurs modalités concrètes. Il est particulièrement inadéquat pour s'occuper de la culture populaire, c'est-à-dire, des pratiques culturelles de la majorité de la population. Il exclut en plus diverses manifestations de la communication humaine (par exemple, la communication face à face, ou musicale, entre autres), et il ne prend pas en considération les intérêts et points de vue de grands segments de la population (par exemple, il ignore la perspective du genre).

20 Il est vrai, cependant, qu'une bonne partie de ce que l'on connait en science de la communication est due à cette ligne d'étude dominante qui, en adoptant une perspective dans laquelle le concept d'information joue un rôle central et la détermination des effets, pour les atteindre ou les combattre, représente son premier objectif, a obtenu une grande diffusion parmi les spécialistes et le public non spécialisé. Entre autres, parce qu'elle a introduit dans le milieu scientifique de la communication, et dans la considération 
publique de ces phénomènes, quelques thèmes centraux des sciences sociales, qui ont aussi été étudiés par presque tous les autres courants scientifiques importants pour l'étude de la communication, et qui aujourd'hui, à une époque de changement et d'incertitude, présentent un intérêt tout particulier car la communication, et notamment celle développée à travers de ces puissants moyens techniques-a beaucoup à voir avec le " pouvoir $»^{5}$.

\section{Le tournant culturel}

21 La définition de l'objet d'étude de cette science est donc due, tout spécialement, à ce courant que j'ai préféré dénommer «informationnel», dont la position hégémonique dans l'histoire de la recherche en communication a contribué de manière très remarquable à placer cet objet d'étude dans une position principale dans cette science, même si les diverses approches critiques de la perspective informationnelle qui se sont développées autour des années 60 ont remis cette approche en question. Parmi ces approches, d'ordre plutôt macroscopique, on mentionnera la théorie culturelle développée dans le cadre de l'École de Frankfort et, surtout, les diverses lignes sémiotiques. On a observé d'autres positionnements, plus microscopiques, de la communication, en particulier celles qui ont étudié de près la subjectivité des récepteurs et l'introduction des media dans leur vie quotidienne. Parmi eux, se détache la ligne d'étude qui met l'accent sur les « usages et gratifications »-inscrite au sein du courant informationnel dominant, quoiqu'elle suppose un développement spécial. Différentes raisons (mercantiles, scientifiques) ont imposé la révision de quelques-unes des idées difficiles à soutenir mais solidement établies sur la science de la communication. Il s'ensuit que l'on pourrait constater le rôle actif du récepteur des messages lorsqu'il choisit une série de contenus dans plusieurs media (comment, quand et pourquoi les utilise-t-il ?) et que l'on remettrait en question les explications monocausales si traditionnelles en science de la communication - reconnaissant ainsi la complexité inhérente à n'importe quelle conduite humaine, que l'école systémique surtout a incorporé à l'étude de la communication.

Ces courants critiques appliqués à l'étude de la communication s'inscrivent dans le contexte plus vaste de la série de changements que les sciences humaines et sociales ont connu. Ils ont cherché à articuler des réponses justes aux changements sociaux qui s'accumulaient déjà à cette époque et qui n'ont fait que s'intensifier et s'accélérer dans les décennies suivantes. L'analyse et l'interprétation de ces changements ont abouti à la publication d'une abondante littérature qui parfois cherche des réponses et des voies pour une compréhension aussi étendue que possible (Castells, 1997-98/ Giddens, 1986 et 1995), parfois se centre surtout sur leur dimension culturelle (Berger et Luckmann, 1997/ Gere, 2002), et parfois étudie spécialement le déploiement d'un nouveau système de communication. Mais une bonne partie de ces analystes est d'accord pour remarquer dans ses travaux - qu'ils soient de nature générale ou plus spécifique- le rôle central que jouent actuellement, au sein de ces systèmes, la culture et les processus de communication. Il s'ensuit qu'un nombre croissant de spécialistes est d'accord pour souligner le besoin de rénover les cadres théoriques pour l'étude de la communication, parce que, si le cadre informationnel présente divers problèmes et insuffisances pour comprendre les processus de communication de masse, il est encore moins pertinent pour 
l'étude juste des phénomènes communicationnels les plus caractéristiques de nos jours (Morris et Ogan, $1996: 1$ ).

23 Certes la société actuelle connaît le déploiement d'un nouveau système de communication, mais elle est aussi immergée, pour des raisons que l'on ne peut pas examiner ici, dans un processus continu de recherche de sens et d'identité -aussi bien individuellement que collectivement. Dans l'actuel contexte social, ces raisons entre autres expliquent la nécessaire émergence pour les sciences de la communication et d'autres sciences sociales, de postulats qui ne cherchent pas seulement à obtenir une soidisant objectivation de la réalité -en accord avec le paradigme positiviste-, mais s'intéressent aussi à la subjectivité humaine et aux contextes de la vie quotidienne, et aussi et surtout, à la production de sens. En effet, il est probable qu'outre les contributions décisives dérivées de l'examen de la communication du point de vue du genre (van Zoonen, 1994) et de l'étude des émotions dans la conduite humaine et ses cultures (Stewart, 2001/ Neiva, 200) les modifications les plus importantes sur l'objet d'étude de la science de la communication sont liées à l'irruption du « sens ».

Quoique cette perspective jouisse d'une tradition longue et bien établie dans les sciences sociales (depuis le «verstehen» wébérien) et connaisse quelques timides tentatives d'implantation dans les premiers pas de la recherche en communication, ce ne sera néanmoins que dans la décennie des années $70 \mathrm{du} \mathrm{XX}$ siècle -et quelque peu avant, avec le travail séminal de Berger et Luckmann (1968) - que le sens émergera comme objet déclaré de la recherche scientifique de la communication. Ainsi, les voies principales par lesquelles on a introduit le sens dans l'étude de la communication ont été certaines études qualitatives des textes médiatiques (la sémiotique tout spécialement, mais la narratologie et la rhétorique aussi) et d'autres travaux dans la ligne de la recherche qualitative sur le terrain -ethnographie des producteurs (Tuchman, 1978) et des récepteurs (Morley, 1992). Mais les contributions probablement les plus brillantes à cet égard sont celles obtenues sur la scène polymorphe des "Cultural Studies» britanniques qui, centrées au début sur deux champs interconnectés (le sens et le pouvoir), ont inclus dans la communication une ample gamme d'études qui reposent sur deux grandes idées : l'une, la polysémie inhérente à tout message et l'autre, la relation active des récepteurs avec ces produits culturels - qu'ils sélectionnent, s'approprient et auxquels ils donnent du sens-; ce qui, bien évidemment, équivaut à mettre l'accent sur le pouvoir des récepteurs en opposition à celui que l'on attribuait traditionnellement aux producteurs et aux textes eux-mêmes.

Il est vrai que le terme «sens » (de l'anglais meaning) connait diverses acceptions dans le vocabulaire scientifique, dérivées sans doute des courants divers qui lui donnent un rôle de la plus grande importance dans l'étude de la communication; mais elles sont également liées aux quatre dimensions essentielles que le sens a dans les communications humaines -dénotative, connotative, super-structurelle, existentielle-(Dahlgren, 1998: 47-8). Bien que tous ces spécialistes coïncident, en général, à donner un rôle décisif aux diverses médiations sociales (Martín Barbero, 1998 : 159) dans la construction sociale du sens $^{6}$, nombre d'entre eux prêtent encore une attention trop exclusive- aux aspects subjectifs, idiosyncratiques, et prennent peu en considération les structures et les modèles culturels propres à la société -ainsi que d'autres éléments matériels et idéaux. Mais, indépendamment de meilleurs développements théoriques que l'on doit obtenir encore- surtout ceux liés à l'articulation entre les composants microstructurels et macrostructurels présents dans tout processus de communication- il n'y a aucun doute, 
cependant, que le sens contribue d'une manière décisive à obtenir une définition meilleure de l'objet d'étude propre à cette science, bien que cette définition doive probablement être plurielle ou, pour le moins, plus ouverte que jusqu'à présent, afin qu'elle puisse comprendre les nombreuses manifestations de la communication humaine ainsi que les logiques et cadres théoriques conséquents.

\section{BIBLIOGRAPHIE}

Ang, Ien (1996) : Las guerras de las salas de estar. Nuevas tecnologías, índices de audiencia y tácticas de consumo. Dans Silverstone, Roger et Hirsch, Eric (eds.) : Los efectos de la nueva comunicación. El consumo de la moderna tecnología en el hogar y en la familia. Paidós : Barcelona, pp. 193-212.

Berger, Peter L. et Luckmann, Thomas (1968) : La construcción social de la realidad. Amorrortu : Buenos Aires.

Berger, Peter L. et Luckmann, Thomas (1997) : Modernidad, pluralismo y crisis de sentido. La orientación del hombre moderno. Paidós : Barcelona.

Bueno, Gustavo (1996) : El mito de la cultura. Prensa Ibérica : Barcelona.

Burnett, Robert et Marshall, P. David (2003) : Web Theory. An Introduction. Routledge : Londres.

Castells, Manuel (1997-98) : La era de la información. Economía, sociedad y cultura (3 vols.). Alianza Editorial : Madrid.

Cook, Nicholas (2003) : De Madonna al canto gregoriano. Una muy breve introducción a la música. Alianza Editorial : Madrid (première réimpression).

Dahlgren, Peter (1998) : Meaning and/vs. Information in Media Studies. Dans Loisir et Société, vol. $21, \mathrm{n}^{\mathrm{o}} 1$, pp. 43-62.

de Aguilera, Miguel (2004) : Tomar la cultura popular en serio, dans Comunicación, 2, 147-58.

de Aguilera, Joaquín et de Aguilera, Miguel (1989) : Nueva dimensión de los medios audiovisuales. Mitre : Barcelona.

del Río, Pablo (1996) : Psicología de los medios de comunicación. Síntesis : Madrid.

Gere, Charles (2002) : Digital Culture. Reaction Books : Londres.

Giddens, Anthony (1986) : La constitución de la sociedad. Bases para la teoría de la estructuración. Amorrortu : Buenos Aires.

Giddens, Anthony (1995) : Modernidad e identidad del yo. (El yo y la sociedad en la época contemporánea). Península : Barcelona.

Manaev, Oleg (2003) : Methodological Trends in Communicational Research. Séminaire imparti à l'Université de Málaga.

Martín Barbero, Jesús (1998) : Penser la société à partir de la communication. Dans Loisir et Société, vol. 21, no 1, pp. 145-77. 
Mattelart, Armand (1993) : La comunicación-mundo. Historia de las ideas y de las estrategias. Fundesco : Madrid.

Mattelart, Armand (1995) : La invención de la comunicación. Bosch : Barcelona.

Miège, Bernard (2004) : L'information-communication, objet de connaissance. De Boeck : Issy-lesMoulineaux.

Morley, David (1992) : Television, Audiences and Cultural Studies. Routledge : Londres.

Morris, M. et Ogan, C. (1996) : The Internet as Mass Medium. Dans Journal of Computer Mediated Communication 1 : 4, dans l'URL : http://shum.cc.huji.ac.il/jcmc/vol1/issue4/morris.html.

Neiva, Eduardo (2001) : Rethinking the foundations of culture. Dans James Lull, ed. : Culture in the Communication Age. Routledge : Nueva York, pp. 31-53.

Real, Michael (2001) : Cultural theory in popular culture and media spectacles. Dans James Lull, ed. : Culture in the Communication Age. Routledge : Nueva York, pp. 167-78.

Stewart, Edward C. (2001) : Culture in the mind : on the origin of meaning and emotion. Dans James Lull, ed. : Culture in the Communication Age. Routledge : Nueva York, pp. 9-30.

Thompson, John B. (1998) : Los media y la modernidad. Una teoría de los medios de comunicación. Paidós : Barcelona.

Tuchman, Gaye (1978) : Making News. Routledge : Londres.

van Zoonen, L. (1994) : Feminist Media Studies. Sage : Newbury Park.

Vitalis, André (1999) : Le déni du politique. Dans Proux, Serge et Vitalis, André (dirs.) : Vers une citoyenneté simulé. Médias, réseaux et mondialisation. Apogée : Rennes.

\section{NOTES}

1. Le rôle de la femme dans l'histoire de la musique culte est mineur, réduit quasi exclusivement à l'interprétation de pièces dans l'enceinte domestique, fréquemment devant des proches ou des candidats à le devenir.

2. Tels que la frayeur causée par les horreurs de cette guerre et l'usage que l'on a y fait des média, ou que la division du monde dans deux grandes blocs de pouvoir, dont chacun a attribué à la science un rôle déterminé.

3. Mesurant des éléments objectivables de façon à ce que, avec des fins prédictives, elle explique les phénomènes en observant ses relations causales -même si on étudie essentiellement une ou peu de variables dans ces relations.

4. Ce dernier élément, de moindre importance mais établi dans le cadre de la Modernité, jette aussi les fondements de plusieurs discours - assez fréquemment utopiques, mais aussi souvent logés à l'enseigne du pessimisme.

5. C'est-à-dire, avec la capacité d'influence («pouvoir culturel » ou « symbolique ») qu'elle a sur ceux qui jouissent d'une position de privilège dans une situation d'échange communicationnel avec des personnes isolées, groupes ou grands collectifs de population-; influence qui, assurément, a été partagée dans l'histoire de la recherche de la communication entre les émetteurs (surtout, par les premiers courants d'étude, spécialement par la «théorie de l'aiguille hypodermique »), les contenus (par les diverses lignes qui se sont préoccupées pour le sens des messages, et particulièrement, les sémiotiques) et les récepteurs (avant tout, le courant des « cultural studies »). Egalement, avec la lutte pour le pouvoir entre les différentes classes sociales (notamment les courants généralement réunis sous le paradigme du matérialisme historique) et 
les «négociations » qu'elles mènent en définissant le sens et l'orientation que l'on doit donner aux messages pendant leur production ou réception. De la même manière, avec le pouvoir pour déterminer, en termes moraux, l'orientation idéale que doit suivre aussi bien la population en général que certains de ses segments - par exemple, enfants et jeunes. Et même, la capacité pour établir qui peut accéder, et comment, à la production et diffusion des messages, ou le rapport de ces contenus avec le « pouvoir économique » (Thompson, $1998: 31$ ).

6. Pas uniquement aux messages médiatiques et, encore moins, à un seul des messages, comme quelques-unes des premières formulations théoriques pour l'étude de la communication l'ont proposé.

\section{RÉSUMÉS}

Nos activités communicationnelles sont conditionnées par le contexte dans lequel elles se développent. La recherche en communication, alors, n'a pu éviter l'influence de divers éléments de type « matériel » et « idéal » qui déterminent les contextes dans lesquels elle s'est développée. Parmi ces éléments, on inclut les manières de définir et de penser la communication, les différentes orientations scientifiques et les objets d'études correspondants. Cet article traite particulièrement de quelques éléments de type «idéal » : les «mythes de la communication et la culture » qui ont contribué à établir des cadres théoriques sur la communication en précisant un objet d'étude. Bien que cet objet d'étude concerne les développements les plus récents, il existe aussi d'autres modèles théoriques, dans un cadre historique soumis aux changements, centrés sur un objet d'étude sûrement plus approprié à comprendre les communications.

As the historical subjects that we are, the different communication activities that we carry out (including those linked to scientific research) are often to a considerable extent conditioned by the context in which they are developed. Communication research therefore, has been unable to distance itself from the influence of the 'material' and 'ideal' type of different elements appearing in those contexts which have been developed. Among those elements we can include the different ways of dealing with communication which, based on certain scientific tendencies, can include both its own defintion and its object of study. This article gives special attention to some ideal elements within this context (the 'myths of communication and culture') which have contributed to establishing theoretical boundaries of communication by establishing an object of study which by projecting itself onto the different forms of human communication, making the due comprehension of some of the more significant forms of communication more difficult since they could appear more obscure.

\section{INDEX}

Mots-clés : modèles de la communication, théorie, épistémologie, culture 


\section{AUTEUR}

\section{MIGUEL DE AGUILERA}

Miguel de Aguilera est docteur en Sociologie et Titulaire de la Chaire de Communication Audiovisuelle et Publicité à l'Université de Málaga, où il enseigne la théorie et la recherche en communication et quelques formes de culture populaire. Sa recherche se concentre sur la relation des jeunes avec la communication et son impact sur les cultures et identités juvéniles, s'occupant de phénomènes tels que les jeux vidéo et la musique ; cette recherche, d'autre part, s'étend aux applications pratiques, comme ces études jeunesse, cultures, pratiques de risque - sont appliquées au domaine de la communication pour la santé. Mail : deaguilera@uma.es 